\title{
A TOPOLOGICAL APPROACH TO THE EXISTENCE AND MULTIPLICITY OF POSITIVE SOLUTIONS OF $(p, q)$-LAPLACIAN SYSTEMS
}

\author{
GENNARO INFANTE, MATEUSZ MACIEJEWSKI, AND RADU PRECUP
}

\begin{abstract}
In this paper we develop a new theory for the existence, localization and multiplicity of positive solutions for a class of non-variational, quasilinear, elliptic systems. In order to do this, we provide a fairly general abstract framework for the existence of fixed points of nonlinear operators acting on cones that satisfy an inequality of Harnack type. Our methodology relies on fixed point index theory. We also provide a non-existence result and an example to illustrate the theory.
\end{abstract}

\section{INTRODUCTION}

In this paper we develop a new theory for the existence, localization and multiplicity of nonnegative weak solutions to the following Dirichlet problem for $(p, q)$-Laplacian systems

$$
\begin{cases}-\Delta_{p} u=f(x, u, v) & \text { in } \Omega, \\ -\Delta_{q} v=g(x, u, v) & \text { in } \Omega, \\ u, v=0 & \text { on } \partial \Omega\end{cases}
$$

where $\Omega \subset \mathbb{R}^{n}$ is a bounded domain of class $C^{1, \gamma}$ for some $\gamma \in(0,1), f, g: \bar{\Omega} \times \mathbb{R}_{+}^{2} \rightarrow \mathbb{R}_{+}$ are continuous functions, and $p, q>2 n /(n+1)$.

The existence of positive solutions of these types of problems has been investigated by means of different methodologies. The most common approach is topological, for example Schauder fixed point theorem was used in [12, Schaefer fixed point theorem was employed in [14], Leray-Shauder degree theory was exploited in [4, 5, 23], fixed point index on cones was applied in [16, 22] and continuation methods were used in [3]. Variational methods, making use of the Nehari manifold, have been used in [1, 20], super and subsolution methods were applied in [7, 11] and monotone techniques in [9].

The multiplicity of solutions was studied in [1, 20, 23], nonexistence was investigated in [5, 22, 23], a priori estimates were given in [3, 23], regularity results were obtained in [7] and qualitative properties of the solutions have been studied in [17].

Localization results have been given in [16], where the authors proved the existence of one positive solution in the case $p=q$, and in [4, where the authors dealt with the existence of radially symmetric solutions in a ball.

Here we develop a new method that deals with the existence, localization and multiplicity of solutions in cones, for systems of two (or more) abstract equations. This method

2010 Mathematics Subject Classification. Primary 35J57, secondary 35B09, 35B45, 35D30, 35J92, $47 \mathrm{H} 10$.

Key words and phrases. Weak Harnack inequality, fixed point index, $p$-Laplace operator, quasilinear elliptic system, positive weak solution, cone, multiplicity, nonexistence. 
can be applied, as a special case, to deal with the existence of positive solutions of the system (1.1). Our approach is purely topological and is based on an abstract Harnacktype inequality and on the fixed point index theory. In order to do this, we fully exploit the recent theory developed by Precup in [18, 19] for the case of one equation, where the author has obtained some Krasnosel'skii-type results. We remark that our results are not a trivial extension of the ones in [18, 19] to the case of systems. In fact, we fully benefit of the richer structure of the system and we improve the theory, even in the case of one equation, by allowing better constants and a more precise localization of the solutions.

In the case of the system 1.1 we obtain existence, localization, multiplicity and nonexistence of positive weak solutions. We also provide an example to illustrate the theoretical results.

Our results are new and improve and complement earlier ones in literature.

\section{Operator equations on Cartesian products}

Let $X_{i}(i=1,2)$ be Banach spaces with norms $|\cdot|_{i}$ ordered by cones $K_{i}^{0}$ and let $\|\cdot\|_{i}$ be seminorms on $X_{i}$. Denote by $\leq_{i}$ the partial order relation associated with $K_{i}^{0}$. Assume that both norms and seminorms are monotone, i.e.

$$
0 \leq_{i} u \leq_{i} v \text { implies }|u|_{i} \leq|v|_{i} \text { and }\|u\|_{i} \leq\|v\|_{i}
$$

for $u, v \in X_{i}$. In what follows, for simplicity, we shall use the same symbols $|\cdot|,\|\cdot\|, \leq$ to denote $|\cdot|_{i},\|\cdot\|_{i}, \leq_{i}$ for both $i=1,2$.

Let $\chi_{i} \in K_{i}^{0}$ be fixed such that $\left\|\chi_{i}\right\|>0$. Define the cones $K_{i} \subset K_{i}^{0}$ by the formula

$$
K_{i}:=\left\{u \in K_{i}^{0}: u \geq\|u\| \chi_{i}\right\}
$$

and assume that there exist points inside them with positive seminorms, which is equivalent to the assumption $\left\|\chi_{i}\right\| \leq 1$. Hence, we can choose

$$
\phi_{i} \in K_{i}, \quad\left|\phi_{i}\right|=1, \quad\left\|\phi_{i}\right\|>0 .
$$

In particular we may take

$$
\phi_{i}=\frac{\chi_{i}}{\left|\chi_{i}\right|}
$$

Let us observe that the seminorm $\|\cdot\|_{i}$ is continuous in $K_{i}$ with respect to the topology induced by the norm $|\cdot|_{i}$, since one has:

$$
u \geq\|u\| \chi_{i}, u \in K_{i} \Longrightarrow\|u\| \leq \frac{1}{\left|\chi_{i}\right|}|u|, u \in K_{i} .
$$

In what follows by compactness of a continuous operator we mean the relative compactness of its range. By complete continuity of a continuous operator we mean the relative compactness of the image of every bounded set of the domain.

We set

$$
X:=X_{1} \times X_{2}, \quad K:=K_{1} \times K_{2},
$$

and we seek the fixed points of a completely continuous operator

$$
N=\left(N_{1}, N_{2}\right): K \rightarrow K,
$$


that is $(u, v) \in K$ with $N(u, v)=(u, v)$. Note that the cone-invariance of $N$ is equivalent to the fact that the operators $N_{i}$ satisfy an abstract weak Harnack inequality of the type

$$
N_{i}(u, v) \geq\left\|N_{i}(u, v)\right\| \chi_{i}, \quad(i=1,2) .
$$

We shall discuss not only existence, but also localization and multiplicity of solutions of the nonlinear equation $N(u, v)=(u, v)$. In order to do this, we utilize the Granas fixed point index, $\operatorname{ind}_{C}(f, U)$ (for more information on the index and its applications we refer the reader to $[\underline{6}, 10]$ ).

The next Proposition describes some of the useful properties of the index, for details see Theorem 6.2, Chapter 12 of [10].

Proposition 2.1. Let $C$ be a closed convex subset of a Banach space, $U \subset C$ be open in $C$ and $f: \bar{U} \rightarrow C$ be a compact map with no fixed points on the boundary $\partial U$ of $U$. Then the fixed point index has the following properties:

(i) (Existence) If $\operatorname{ind}_{C}(f, U) \neq 0$ then $\operatorname{fix}(f) \neq \emptyset$, where fix $f=\{x \in \bar{U}: f(x)=x\}$.

(ii) (Additivity) If fix $f \subset U_{1} \cup U_{2} \subset U$ with $U_{1}, U_{2}$ open in $C$ and disjoint, then

$$
\operatorname{ind}_{C}(f, U)=\operatorname{ind}_{C}\left(f, U_{1}\right)+\operatorname{ind}_{C}\left(f, U_{2}\right) .
$$

(iii) (Homotopy invariance) If $h: \bar{U} \times[0,1] \rightarrow C$ is a compact homotopy such that $h(u, t) \neq u$ for $u \in \partial U$ and $t \in[0,1]$ then

$$
\operatorname{ind}_{C}(h(., 0), U)=\operatorname{ind}_{C}(h(., 1), U) .
$$

(iv) (Normalization) If $f$ is a constant map, with $f(u)=u_{0}$ for every $u \in \bar{U}$, then

$$
\operatorname{ind}_{C}(f, U)= \begin{cases}1, & \text { if } u_{0} \in U \\ 0, & \text { if } u_{0} \notin \bar{U} .\end{cases}
$$

In particular, $\operatorname{ind}_{C}(f, C)=1$ for every compact function $f: C \rightarrow C$, since $f$ is homotopic to any $u_{0} \in C$, by the convexity of $C\left(\right.$ take $\left.h(u, t)=t f(u)+(1-t) u_{0}\right)$.

2.1. Solutions with at least one nonzero component. We begin with four theorems on the existence and localization of one solution of the operator equation $N(u, v)=(u, v)$. The first two ones assume that the operator $N$ leaves invariant the set

$$
C=\left\{(u, v) \in K:|u| \leq R_{1},|v| \leq R_{2}\right\},
$$

for some fixed numbers $R_{1}, R_{2}$.

Theorem 2.2. Assume that there exist numbers $r_{i}>0$ and $R_{i}>0$ with

$$
r_{i}<\left\|\phi_{i}\right\|\left\|\chi_{i}\right\| R_{i} \quad(i=1,2),
$$

such that

$$
\inf _{\substack{(u, v) \in C \\\|u\|=r_{1},\|v\| \leq r_{2}}}\left\|N_{1}(u, v)\right\| \geq \frac{r_{1}}{\left\|\chi_{1}\right\|}, \quad \inf _{\substack{(u, v) \in C \\\|u\| \leq r_{1},\|v\|=r_{2}}}\left\|N_{2}(u, v)\right\| \geq \frac{r_{2}}{\left\|\chi_{2}\right\|},
$$

and

$$
\sup _{(u, v) \in C}\left|N_{i}(u, v)\right| \leq R_{i} \quad(i=1,2) .
$$


Then $N$ has at least one fixed point $(u, v) \in K$ such that $|u| \leq R_{1},|v| \leq R_{2}$ and either $\|u\| \geq r_{1}$ or $\|v\| \geq r_{2}$.

Proof. The assumption (2.5) implies that $N(C) \subset C$. Therefore, by Proposition 2.1. $\operatorname{ind}_{C}(N, C)=1$. Let

$$
U:=\left\{(u, v) \in C:\|u\|<r_{1},\|v\|<r_{2}\right\} .
$$

The boundary $\partial U$ of $U$ with respect to $C$ is equal to $\partial U=A_{1} \cup A_{2}$, where

$$
\begin{aligned}
& A_{1}=\left\{(u, v) \in C:\|u\|=r_{1},\|v\| \leq r_{2}\right\}, \\
& A_{2}=\left\{(u, v) \in C:\|u\| \leq r_{1},\|v\|=r_{2}\right\} .
\end{aligned}
$$

If there is a fixed point $(u, v)$ of $N$ on $\partial U$, then $(u, v)$ satisfies the assertion. If not, the indices $\operatorname{ind}_{C}(N, U)$ and $\operatorname{ind}_{C}(N, C \backslash \bar{U})$ are well defined and their sum, by the additivity property of the index, is equal to one. Therefore, it suffices to prove that $\operatorname{ind}_{C}(N, U)=0$. Take $h=\left(R_{1} \phi_{1}, R_{2} \phi_{2}\right) \in C$ and consider the homotopy $H: C \times[0,1] \rightarrow C$,

$$
H(u, v, t):=t h+(1-t) N(u, v) .
$$

We claim that $H$ is fixed point free on $\partial U$. Since

$$
\begin{aligned}
\left\|R_{1} \phi_{1}\right\| & =R_{1}\left\|\phi_{1}\right\| \geq R_{1}\left\|\phi_{1}\right\|\left\|\chi_{1}\right\|>r_{1}, \\
\left\|R_{2} \phi_{2}\right\| & =R_{2}\left\|\phi_{2}\right\| \geq R_{2}\left\|\phi_{2}\right\|\left\|\chi_{2}\right\|>r_{2},
\end{aligned}
$$

we have that $(u, v) \neq h=H(u, v, 1)$ for all $(u, v) \in \partial U$. It remains to show that $H(u, v, t) \neq(u, v)$ for $(u, v) \in \partial U$ and $t \in(0,1)$. Assume the contrary. Then there exists $(u, v) \in A_{1} \cup A_{2}$ and $t \in(0,1)$ such that

$$
(u, v)=t h+(1-t) N(u, v) .
$$

Suppose that $(u, v) \in A_{1}$. Then, exploiting the first coordinate of the equation (2.7), we obtain that

$$
\begin{aligned}
u & =t R_{1} \phi_{1}+(1-t) N_{1}(u, v) \geq t R_{1}\left\|\phi_{1}\right\| \chi_{1}+(1-t)\left\|N_{1}(u, v)\right\| \chi_{1} \\
& \geq\left(t R_{1}\left\|\phi_{1}\right\|+(1-t) \inf _{\substack{(u, v) \in C \\
\|u\|=r_{1},\|v\| \leq r_{2}}}\left\|N_{1}(u, v)\right\|\right) \chi_{1} \\
& \geq\left(t R_{1}\left\|\phi_{1}\right\|+(1-t) \frac{r_{1}}{\left\|\chi_{1}\right\|}\right) \chi_{1} .
\end{aligned}
$$

Using the monotonicity of $\|\cdot\|$ and $(2.3)$ we obtain that

$$
\begin{aligned}
r_{1} & \geq\left(t R_{1}\left\|\phi_{1}\right\|+(1-t) \frac{r_{1}}{\left\|\chi_{1}\right\|}\right)\left\|\chi_{1}\right\| \\
& =t R_{1}\left\|\phi_{1}\right\|\left\|\chi_{1}\right\|+(1-t) r_{1}>t r_{1}+(1-t) r_{1}=r_{1},
\end{aligned}
$$

which is impossible. Similarly we derive a contradiction if $(u, v) \in A_{2}$.

By the homotopy invariance of the index we obtain that $\operatorname{ind}_{C}(N, U)=\operatorname{ind}_{C}(h, U)$. From 2.6 we have $h \notin \bar{U}$, hence $\operatorname{ind}_{C}(N, U)=\operatorname{ind}_{C}(h, U)=0$, as we wished. 
Remark 2.3. We observe that, using the relation (2.2), a lower bound for the solution in terms of the seminorm provides a lower bound for the norm of the solution, namely

$$
\|u\| \geq r_{1} \Longrightarrow|u| \geq r_{1}\left|\chi_{1}\right| \text {. }
$$

In the next result we replace, in the spirit of Lemma 4 of [13], the assumption (2.4) with a different one. The two conditions are not comparable and are used, in a combined way, in Theorem 2.14 .

Theorem 2.4. Assume that there exist numbers $r_{i}>0$ and $R_{i}>0$ with

$$
r_{i}<\left\|\phi_{i}\right\|\left\|\chi_{i}\right\| R_{i} \quad(i=1,2),
$$

such that

$$
\sup _{(u, v) \in C}\left|N_{i}(u, v)\right| \leq R_{i} \quad(i=1,2)
$$

and

$$
\inf _{(u, v) \in A}\left\|N_{1}(u, v)\right\| \geq r_{1} \quad \text { or } \inf _{(u, v) \in A}\left\|N_{2}(u, v)\right\| \geq r_{2},
$$

where $A$ is a subset of the set

$$
U=\left\{(u, v) \in C:\|u\|<r_{1},\|v\|<r_{2}\right\} .
$$

Then $N$ has at least one fixed point $(u, v) \in K$ such that $|u| \leq R_{1},|v| \leq R_{2}$ and $(u, v) \notin A$.

Proof. Since $N$ is a completely continuous mapping in the bounded closed convex set $C$, by Schauder's fixed point theorem, it possesses a fixed point $(u, v) \in C$. We now show that the fixed point is not in $A$. Suppose on the contrary that $(u, v)=N(u, v)$ and $(u, v) \in A$. Suppose that the first inequality from (2.11) is satisfied. Then

$$
r_{1}>\|u\|=\left\|N_{1}(u, v)\right\| \geq r_{1},
$$

which is impossible. Similarly we arrive at a contradiction, if the second inequality from (2.11) is satisfied.

The next theorems do not assume the invariance condition $N(C) \subset C$ and use instead Leray-Schauder type conditions. The first result requires the Leray-Schauder condition componentwise.

Theorem 2.5. Assume that there exist numbers $r_{i}>0$ and $R_{i}>0$ with

$$
r_{i}<\left\|\phi_{i}\right\|\left\|\chi_{i}\right\| R_{i} \quad(i=1,2),
$$

such that the strengthened condition (2.4):

$$
\begin{aligned}
& \inf _{\substack{(u, v) \in C \\
\|u\|=r_{1},\|v\| \leq r_{2}}}\left(\max \left\{\frac{\left|N_{1}(u, v)\right|}{R_{1}}, 1\right\}\right)^{-1}\left\|N_{1}(u, v)\right\| \geq \frac{r_{1}}{\left\|\chi_{1}\right\|}, \\
& \inf _{\substack{(u, v) \in C \\
\|u\| \leq r_{1},\|v\|=r_{2}}}\left(\max \left\{\frac{\left|N_{2}(u, v)\right|}{R_{2}}, 1\right\}\right)^{-1}\left\|N_{2}(u, v)\right\| \geq \frac{r_{2}}{\left\|\chi_{2}\right\|},
\end{aligned}
$$


and the weakened condition 2.5):

$$
|u|=R_{1},|v|<R_{2} \quad \text { implies } N(u, v) \neq(\lambda u, v), \text { for all } \lambda>1 ;
$$

$$
\begin{aligned}
& |u|<R_{1},|v|=R_{2} \quad \text { implies } N(u, v) \neq(u, \lambda v), \text { for all } \lambda>1 ; \\
& |u|=R_{1},|v|=R_{2} \quad \text { implies } N(u, v) \neq\left(\lambda_{1} u, \lambda_{2} v\right), \text { for all } \lambda_{1}, \lambda_{2} \geq 1 \text { with } \lambda_{1} \lambda_{2}>1,
\end{aligned}
$$

hold. Then $N$ has at least one fixed point $(u, v) \in K$ such that $|u| \leq R_{1},|v| \leq R_{2}$ and either $\|u\| \geq r_{1}$ or $\|v\| \geq r_{2}$.

Proof. Consider the retraction $\pi: K \rightarrow C, \pi(u, v)=\left(\pi_{1}(u), \pi_{2}(v)\right)$, where

$$
\pi_{i}: K_{i} \rightarrow C_{i}:=\left\{u \in K_{i}:|u| \leq R_{i}\right\}, \quad \pi_{i}(u)= \begin{cases}u & \text { if }|u| \leq R_{i} \\ \frac{R_{i}}{|u|} u & \text { if }|u| \geq R_{i} .\end{cases}
$$

Now we define the operator $\tilde{N}$ by the formula

$$
\tilde{N}(u, v)=\pi(N(u, v))=\left(\pi_{1}\left(N_{1}(u, v)\right), \pi_{2}\left(N_{2}(u, v)\right)\right) .
$$

Then $\tilde{N}(C) \subset C$, i.e. $\tilde{N}$ satisfies (2.5). Now, let $(u, v) \in C$ be such that $\|u\|=r_{1},\|v\| \leq$ $r_{2}$. Observe that

$$
\tilde{N}_{1}(u, v)=\pi_{1}\left(N_{1}(u, v)\right)=\left(\max \left\{\frac{\left|N_{1}(u, v)\right|}{R_{1}}, 1\right\}\right)^{-1} N_{1}(u, v),
$$

which in view of $(2.13)$ shows that

$$
\left\|\tilde{N}_{1}(u, v)\right\| \geq \frac{r_{1}}{\left\|\chi_{1}\right\|}
$$

A similar estimate holds for $\tilde{N}_{2}$, which shows that $\tilde{N}$ satisfies 2.4 . By Theorem 2.2 we obtain a fixed point $(u, v)$ of $\tilde{N}$ in the set $C \backslash U$. Therefore $\pi(N(u, v))=(u, v)$. If $\left|N_{1}(u, v)\right| \leq R_{1}$, then $\pi_{1}\left(N_{1}(u, v)\right)=N_{1}(u, v)$ and so $N_{1}(u, v)=u$. Similarly, if $\left|N_{2}(u, v)\right| \leq R_{2}$, then $N_{2}(u, v)=v$, and the proof is complete. Otherwise, we have $\left|N_{1}(u, v)\right|>R_{1}$ or $\left|N_{2}(u, v)\right|>R_{2}$. Assume $\left|N_{1}(u, v)\right|>R_{1}$. Then

$$
\pi_{1}\left(N_{1}(u, v)\right)=\frac{R_{1}}{\left|N_{1}(u, v)\right|} N_{1}(u, v)=u .
$$

It follows that $N_{1}(u, v)=\frac{\left|N_{1}(u, v)\right|}{R_{1}} u$ and $|u|=R_{1}$ which, in view of the first implication from (2.14), is impossible if $|v|<R_{2}$. Hence $|v|=R_{2}$, which implies that $N_{2}(u, v)=\lambda_{2} v$ for some $\lambda_{2} \geq 1$. Since $N_{1}(u, v)=\lambda_{1} u$, where $\lambda_{1}=\frac{\left|N_{1}(u, v)\right|}{R_{1}}>1$ and $|u|=R_{1}$, we contradict the third condition from (2.14). Thus the inequality $\left|N_{1}(u, v)\right|>R_{1}$ is not possible. By symmetry, the inequality $\left|N_{2}(u, v)\right|>R_{2}$ is also not possible. Consequently, $\pi(N(u, v))=N(u, v)=(u, v)$.

Remark 2.6. Notice that under condition (2.5), (2.14) is satisfied and (2.13) reduces to (2.4).

If instead of the retraction $\pi$, we consider the retraction $\rho: K \rightarrow C$ given by

$$
\rho(u, v)=\left(\max \left\{\frac{|u|}{R_{1}}, \frac{|v|}{R_{2}}, 1\right\}\right)^{-1}(u, v)
$$


we obtain the following existence theorem under the Leray-Schauder condition acting this time, uniformly on the two components $u, v$.

Theorem 2.7. Assume that there exist numbers $r_{i}>0$ and $R_{i}>0$ with

$$
r_{i}<\left\|\phi_{i}\right\|\left\|\chi_{i}\right\| R_{i} \quad(i=1,2),
$$

such that the strengthened condition (2.4):

$$
\begin{aligned}
& \inf _{\substack{(u, v) \in C \\
\|u\|=r_{1},\|v\| \leq r_{2}}}\left(\max \left\{\frac{\left|N_{1}(u, v)\right|}{R_{1}}, \frac{\left|N_{2}(u, v)\right|}{R_{2}}, 1\right\}\right)^{-1}\left\|N_{1}(u, v)\right\| \geq \frac{r_{1}}{\left\|\chi_{1}\right\|}, \\
& \inf _{\substack{(u, v) \in C \\
\|u\| \leq r_{1},\|v\|=r_{2}}}\left(\max \left\{\frac{\left|N_{1}(u, v)\right|}{R_{1}}, \frac{\left|N_{2}(u, v)\right|}{R_{2}}, 1\right\}\right)^{-1}\left\|N_{2}(u, v)\right\| \geq \frac{r_{2}}{\left\|\chi_{2}\right\|},
\end{aligned}
$$

and the weakened condition 2.5$)$ :

$$
N(u, v) \neq \lambda(u, v), \quad \text { for }(u, v) \in \partial C, \lambda>1,
$$

hold. Then $N$ has at least one fixed point $(u, v) \in K$ such that $|u| \leq R_{1},|v| \leq R_{2}$ and either $\|u\| \geq r_{1}$ or $\|v\| \geq r_{2}$.

Proof. The assumption 2.16) guarantees that the mapping $\bar{N}(u, v)=\rho(N(u, v))$, that has the property $\bar{N}(C) \subset C$, also satisfies condition 2.4. By Theorem 2.2, $\bar{N}$ has a fixed point $(u, v)$ in $C \backslash U$. If $N(u, v) \in C$, then $\rho(N(u, v))=N(u, v)$ and we are done. Otherwise, $\left|N_{1}(u, v)\right|>R_{1}$ or $\left|N_{2}(u, v)\right|>R_{2}$, and then $\lambda:=\max \left\{\frac{\left|N_{1}(u, v)\right|}{R_{1}}, \frac{\left|N_{2}(u, v)\right|}{R_{2}}, 1\right\}>$ $1, N(u, v)=\lambda(u, v)$ and $(u, v) \in \partial C$, which is excluded by (2.17).

Remark 2.8. Note that, in the case of one equation, the retractions $\pi$ and $\rho$ coincide with the one used in [18, Theorem 2.1], but are used differently.

2.2. Solutions with both nonzero components. In the previous results one of the components of the solution can be 0 . The next three theorems avoid this situation.

Theorem 2.9. Assume that there exist numbers $r_{i}>0$ and $R_{i}>0$ with

$$
r_{i}<\left\|\phi_{i}\right\|\left\|\chi_{i}\right\| R_{i} \quad(i=1,2),
$$

such that

$$
\inf _{\substack{(u, v) \in C \\\|u\|=r_{1},\|v\| \geq r_{2}}}\left\|N_{1}(u, v)\right\| \geq \frac{r_{1}}{\left\|\chi_{1}\right\|}, \quad \inf _{\substack{(u, v) \in C \\\|u\| \geq r_{1},\|v\|=r_{2}}}\left\|N_{2}(u, v)\right\| \geq \frac{r_{2}}{\left\|\chi_{2}\right\|},
$$

and

$$
\sup _{(u, v) \in C}\left|N_{i}(u, v)\right| \leq R_{i} \quad(i=1,2) .
$$

Then $N$ has at least one fixed point $(u, v) \in K$ such that $|u| \leq R_{1},|v| \leq R_{2},\|u\| \geq r_{1}$ and $\|v\| \geq r_{2}$. 
Proof. As before, the assumption 2.20 implies that $N(C) \subset C$. Thus, $\operatorname{ind}_{C}(N, C)=1$. In order to finish the proof, it is sufficient to show that $\operatorname{ind}_{C}(N, V)=0$, where

$$
V:=\left\{(u, v) \in C:\|u\|<r_{1} \text { or }\|v\|<r_{2}\right\} .
$$

We have $\partial V=B_{1} \cup B_{2}$, where

$$
\begin{aligned}
& B_{1}=\left\{(u, v) \in C:\|u\|=r_{1},\|v\| \geq r_{2}\right\} \\
& B_{2}=\left\{(u, v) \in C:\|u\| \geq r_{1},\|v\|=r_{2}\right\}
\end{aligned}
$$

If $N$ has a fixed point $(u, v) \in \partial V$, we are finished. Thus, assume that $N(u, v) \neq(u, v)$ for $(u, v) \in \partial V$, and consider the same homotopy as in the proof of Theorem 2.2, that is

$$
H(u, v, t)=t h+(1-t) N(u, v) \text {, where } h=\left(R_{1} \phi_{1}, R_{2} \phi_{2}\right) .
$$

We claim that $H$ is fixed point free on $\partial V$. From the previous assumption, $H(u, v, 0) \neq$ $(u, v)$ for $(u, v) \in \partial V$. Also, as in the proof of Theorem 2.2, $H(u, v, 1)=h \neq(u, v)$ on $\partial V$. It remains to show that $H(u, v, t) \neq(u, v)$ for $(u, v) \in \partial V$ and $t \in(0,1)$. Assume the contrary. Then, there exists $(u, v) \in B_{1} \cup B_{2}$ and $t \in(0,1)$ such that

$$
(u, v)=t h+(1-t) N(u, v) .
$$

Suppose that $(u, v) \in B_{1}$. Then, exploiting the first coordinate of the equation (2.21), we obtain that

$$
u=t R_{1} \phi_{1}+(1-t) N_{1}(u, v) \geq t R_{1}\left\|\phi_{1}\right\| \chi_{1}+(1-t)\left\|N_{1}(u, v)\right\| \chi_{1}
$$

$$
\geq\left(t R_{1}\left\|\phi_{1}\right\|+(1-t) \inf _{\substack{(u, v) \in C \\\|u\|=r_{1},\|v\| \geq r_{2}}}\left\|N_{1}(u, v)\right\|\right) \chi_{1} \geq\left(t R_{1}\left\|\phi_{1}\right\|+(1-t) \frac{r_{1}}{\left\|\chi_{1}\right\|}\right) \chi_{1}
$$

thus, as in the proof of Theorem 2.2, we obtain the contradiction $r_{1}>r_{1}$. The case $(u, v) \in B_{2}$ is similar. Therefore $\operatorname{ind}_{C}(N, V)=\operatorname{ind}_{C}(h, V)=0$ since in view of (2.6), $h \notin \bar{V}$.

Theorem 2.9 can be generalized in the spirit of Theorems 2.5 and 2.7 .

Theorem 2.10. Assume that there exist numbers $r_{i}>0$ and $R_{i}>0$ with

$$
r_{i}<\left\|\phi_{i}\right\|\left\|\chi_{i}\right\| R_{i} \quad(i=1,2),
$$

such that

$$
\begin{aligned}
& \inf _{\substack{(u, v) \in C \\
\|u\|=r_{1},\|v\| \geq r_{2}}}\left(\max \left\{\frac{\left|N_{1}(u, v)\right|}{R_{1}}, 1\right\}\right)^{-1}\left\|N_{1}(u, v)\right\| \geq \frac{r_{1}}{\left\|\chi_{1}\right\|}, \\
& \inf _{\substack{(u, v) \in C \\
\|u\| \geq r_{1},\|v\|=r_{2}}}\left(\max \left\{\frac{\left|N_{2}(u, v)\right|}{R_{2}}, 1\right\}\right)^{-1}\left\|N_{2}(u, v)\right\| \geq \frac{r_{2}}{\left\|\chi_{2}\right\|},
\end{aligned}
$$


and

$$
|u|=R_{1},|v|<R_{2} \quad \text { implies } N(u, v) \neq(\lambda u, v), \text { for all } \lambda>1 ;
$$

$$
\begin{aligned}
& |u|<R_{1},|v|=R_{2} \quad \text { implies } N(u, v) \neq(u, \lambda v), \text { for all } \lambda>1 ; \\
& |u|=R_{1},|v|=R_{2} \quad \text { implies } \quad N(u, v) \neq\left(\lambda_{1} u, \lambda_{2} v\right) \text {, for all } \lambda_{1}, \lambda_{2} \geq 1 \text { with } \lambda_{1} \lambda_{2}>1 .
\end{aligned}
$$

Then $N$ has at least one fixed point $(u, v) \in K$ such that $|u| \leq R_{1},|v| \leq R_{2},\|u\| \geq r_{1}$ and $\|v\| \geq r_{2}$.

Proof. Define the retractions $\pi_{i}$ and the operator $\tilde{N}$ as in the proof of Theorem 2.5. Then $\tilde{N}(C) \subset C$, i.e. $\tilde{N}$ satisfies 2.20). Now, let $(u, v) \in C$ be such that $\|u\|=r_{1},\|v\| \geq r_{2}$. Observe that

$$
\tilde{N}_{1}(u, v)=\pi_{1}\left(N_{1}(u, v)\right)=\left(\max \left\{\frac{\left|N_{1}(u, v)\right|}{R_{1}}, 1\right\}\right)^{-1} N_{1}(u, v),
$$

which shows that

$$
\left\|\tilde{N}_{1}(u, v)\right\| \geq \frac{r_{1}}{\left\|\chi_{1}\right\|}
$$

A similar estimate holds for $\tilde{N}_{2}$ which shows that $\tilde{N}$ satisfies 2.19 . By Theorem 2.9 we obtain a fixed point $(u, v)$ of $\tilde{N}$ in the set $C \backslash V$. Therefore $\pi(N(u, v))=(u, v)$. In the proof of Theorem 2.5 it was shown that 2.25) yields $N(u, v)=(u, v)$.

Similarly, using the method presented in the proof of Theorem 2.7 and exploiting Theorem 2.9, we obtain the following fact.

Theorem 2.11. Assume that there exist numbers $r_{i}>0$ and $R_{i}>0$ with

$$
r_{i}<\left\|\phi_{i}\right\|\left\|\chi_{i}\right\| R_{i} \quad(i=1,2),
$$

such that

$$
\begin{aligned}
& \inf _{\substack{(u, v) \in C \\
\|u\|=r_{1},\|v\| \geq r_{2}}}\left(\max \left\{\frac{\left|N_{1}(u, v)\right|}{R_{1}}, \frac{\left|N_{2}(u, v)\right|}{R_{2}}, 1\right\}\right)^{-1}\left\|N_{1}(u, v)\right\| \geq \frac{r_{1}}{\left\|\chi_{1}\right\|}, \\
& \inf _{\substack{(u, v) \in C \\
\|u\| \geq r_{1},\|v\|=r_{2}}}\left(\max \left\{\frac{\left|N_{1}(u, v)\right|}{R_{1}}, \frac{\left|N_{2}(u, v)\right|}{R_{2}}, 1\right\}\right)^{-1}\left\|N_{2}(u, v)\right\| \geq \frac{r_{2}}{\left\|\chi_{2}\right\|},
\end{aligned}
$$

and

$$
N(u, v) \neq \lambda(u, v), \quad \text { for }(u, v) \in \partial C, \lambda>1 .
$$

Then $N$ has at least one fixed point $(u, v) \in K$ such that $|u| \leq R_{1},|v| \leq R_{2},\|u\| \geq r_{1}$ and $\|v\| \geq r_{2}$.

Proof. Let the mapping $\bar{N}$ be defined as in the proof of Theorem 2.7. The assumption 2.27) guarantees that $\bar{N}$, having the property $\bar{N}(C) \subset C$, also satisfies condition 2.19. By Theorem 2.9, $\bar{N}$ has a fixed point $(u, v)$ in $C \backslash V$. It was shown in the proof of Theorem 2.7 that 2.28 implies $N(u, v)=(u, v)$. 
2.3. Multiplicity results. Recall that the seminorms $\|\cdot\|_{i}$ are continuous in $K_{i}$ with respect to the topology induced by $|\cdot|_{i}$, which implies that there exist constants $c_{i}>0$ such that $\|u\|_{i} \leq c_{i}|u|_{i}$ for all $u \in K_{i}$.

Theorem 2.12. Assume that there exist numbers $\rho_{i}, r_{i}, R_{i}$ with

$$
0<c_{i} \rho_{i}<r_{i}<\left\|\phi_{i}\right\|\left\|\chi_{i}\right\| R_{i} \quad(i=1,2),
$$

such that

$$
\begin{gathered}
\inf _{\substack{(u, v) \in C \\
\|u\|=r_{1},\|v\| \geq r_{2}}}\left\|N_{1}(u, v)\right\|>\frac{r_{1}}{\left\|\chi_{1}\right\|}, \quad \inf _{\substack{(u, v) \in C \\
\|u\| \geq r_{1},\|v\|=r_{2}}}\left\|N_{2}(u, v)\right\|>\frac{r_{2}}{\left\|\chi_{2}\right\|}, \\
\sup _{(u, v) \in C}\left|N_{i}(u, v)\right| \leq R_{i} \quad(i=1,2),
\end{gathered}
$$

and

$$
N(u, v) \neq \lambda(u, v), \quad \text { for } \lambda \geq 1 \text { and }\left(|u|=\rho_{1},|v| \leq \rho_{2} \text { or }|u| \leq \rho_{1},|v|=\rho_{2}\right) .
$$

Then $N$ has at least three fixed points $\left(u_{i}, v_{i}\right) \in C(i=1,2,3)$ with

$\left|u_{1}\right|<\rho_{1}, \quad\left|v_{1}\right|<\rho_{2} \quad$ (possibly zero solution)

$\left\|u_{2}\right\|<r_{1}$ or $\left\|v_{2}\right\|<r_{2} ;\left|u_{2}\right|>\rho_{1}$ or $\left|v_{2}\right|>\rho_{2} \quad$ (possibly one solution component zero);

$\left\|u_{3}\right\|>r_{1},\left\|v_{3}\right\|>r_{2}$ (both solution components nonzero).

Proof. Let $V$ be as in the proof of Theorem 2.9. Strict inequalities in 2.30) guarantee that $N$ is fixed point free on $\partial V$. According to the proof of Theorem 2.9 we have $\operatorname{ind}_{C}(N, C)=$ $1, \operatorname{ind}_{C}(N, V)=0$ and therefore by the additivity property, $\operatorname{ind}_{C}(N, C \backslash \bar{V})=1$. Let

$$
W:=\left\{(u, v) \in C:|u|<\rho_{1},|v|<\rho_{2}\right\} .
$$

For every $(u, v) \in \bar{W}$, we have

$$
\|u\| \leq c_{1}|u| \leq c_{1} \rho_{1}<c_{1}\left(\frac{1}{c_{1}} r_{1}\right)=r_{1}
$$

and, similarly, $\|v\|<r_{2}$. Hence $(u, v) \in V$, which proves that $\bar{W} \subset V$. Condition 2.32) shows that $N$ is homotopic with zero on $W$. $\operatorname{Thus}_{\operatorname{ind}}(N, W)=\operatorname{ind}_{C}(0, W)=1$. Then $\operatorname{ind}_{C}(N, V \backslash \bar{W})=0-1=-1$. Consequently, there exist at least three fixed points of $N$, in $W, V \backslash \bar{W}$ and $C \backslash \bar{V}$.

If we assume the following estimates of the $\left\|N_{i}(u, v)\right\|$ :

$$
\inf _{\substack{u, v) \in C \\\|u\|=r_{1}}}\left\|N_{1}(u, v)\right\|>\frac{r_{1}}{\left\|\chi_{1}\right\|}, \inf _{\substack{(u, v) \in C \\\|v\|=r_{2}}}\left\|N_{2}(u, v)\right\|>\frac{r_{2}}{\left\|\chi_{2}\right\|},
$$

then we can obtain a more precise localization for the solution $\left(u_{2}, v_{2}\right)$ in Theorem 2.12 . the Figure 1 illustrates this fact. 

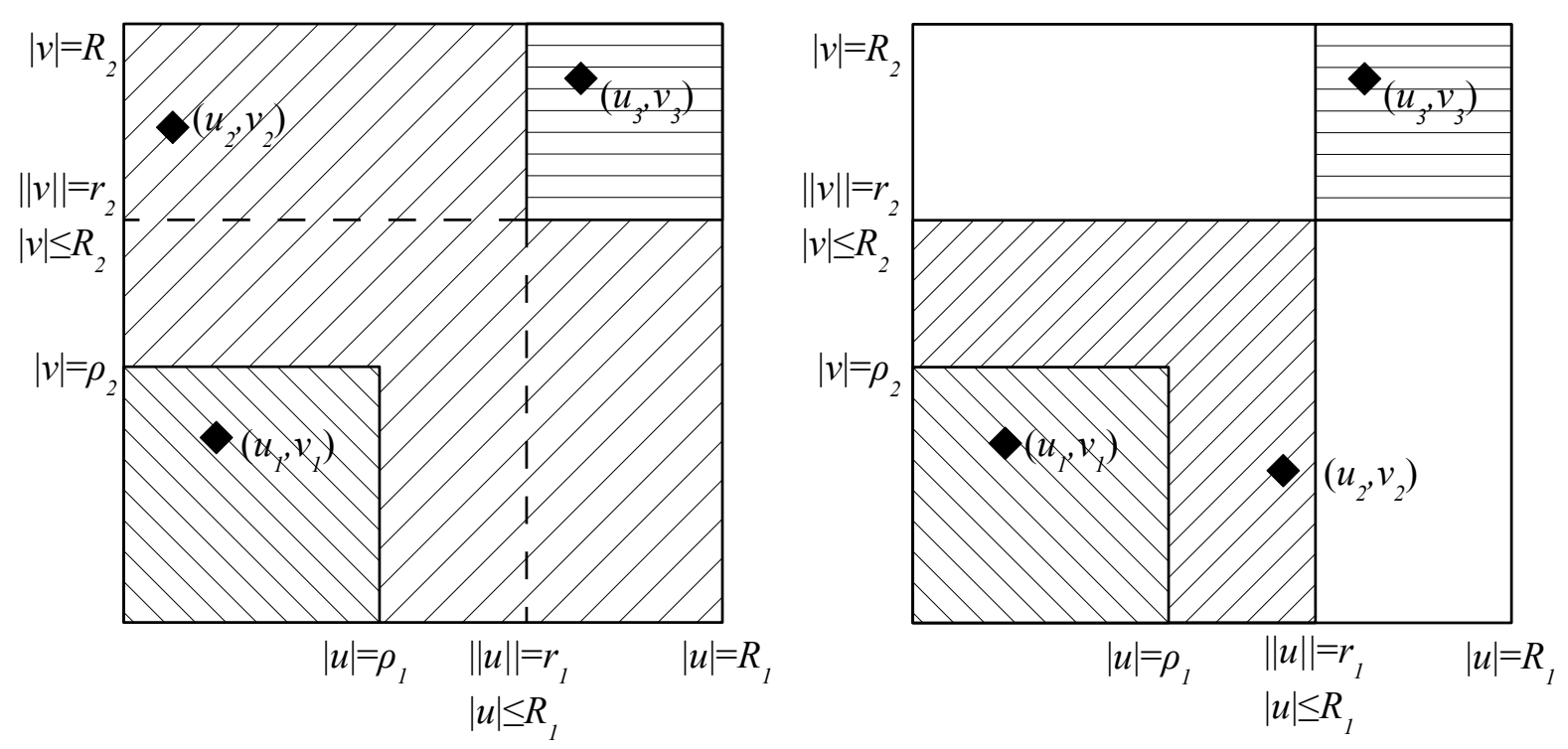

Figure 1. Localization of the three solutions $\left(u_{i}, v_{i}\right)$ from Theorem 2.12 (on the left) and 2.13 (on the right).

Theorem 2.13. Suppose that all the assumptions of Theorem 2.12 are satisfied with the condition 2.30 replaced by 2.33). Then $N$ has at least three fixed points $\left(u_{i}, v_{i}\right) \in C$ $(i=1,2,3)$ with

$\left|u_{1}\right|<\rho_{1}, \quad\left|v_{1}\right|<\rho_{2} \quad$ (possibly zero solution)

$\left\|u_{2}\right\|<r_{1},\left\|v_{2}\right\|<r_{2} ;\left|u_{2}\right|>\rho_{1}$ or $\left|v_{2}\right|>\rho_{2} \quad$ (possibly one solution component zero);

$\left\|u_{3}\right\|>r_{1},\left\|v_{3}\right\|>r_{2}$ (both solution components nonzero).

Proof. The assumption (2.33) implies both (2.4) and (2.19) and that there are no fixed points of $N$ on $\partial U$ and $\partial V$. Hence, as in the proofs of Theorems 2.2 and 2.9, the indices $\operatorname{ind}_{C}(N, U)$ and $\operatorname{ind}_{C}(N, V)$ are well defined and equal 0 . An analysis similar to that in the proof of Theorem 2.12 shows that

$$
\operatorname{ind}_{C}(N, W)=1, \operatorname{ind}_{C}(N, U \backslash \bar{W})=-1, \operatorname{ind}_{C}(N, C \backslash \bar{V})=1,
$$

which completes the proof.

In order to ensure that the solution $\left(u_{1}, v_{1}\right)$ from the theorems above is nonzero, and thereby to obtain three nonzero solutions, we use some additional assumptions on $N$.

Theorem 2.14. Assume that all the conditions of Theorem 2.12 or Theorem 2.13 are satisfied. Consider $0<\varrho_{i}<\left\|\phi_{i}\right\|\left\|\chi_{i}\right\| \rho_{i}, i=1,2$.

(i) If

$$
\inf _{\substack{(u, v) \in K,|u| \leq \rho_{1},|v| \leq \rho_{2} \\\|u\|=\varrho_{1},\|v\| \geq \varrho_{2}}}\left\|N_{1}(u, v)\right\| \geq \frac{\varrho_{1}}{\left\|\chi_{1}\right\|}, \quad \inf _{\substack{(u, v) \in K,|u| \leq \rho_{1},|v| \leq \rho_{2} \\\|u\| \geq \varrho_{1},\|v\|=\varrho_{2}}}\left\|N_{2}(u, v)\right\| \geq \frac{\varrho_{2}}{\left\|\chi_{2}\right\|}
$$

and

$$
\left|N_{i}(u, v)\right| \leq \rho_{i} \text { for }|u| \leq \rho_{1},|v| \leq \rho_{2} \quad(i=1,2),
$$

then we can assume that the solution $\left(u_{1}, v_{1}\right)$ from Theorem 2.12 or 2.13 satisfies $\left\|u_{1}\right\| \geq$ $\varrho_{1}$ and $\left\|v_{1}\right\| \geq \varrho_{2}$; 
(ii) if

$$
\inf _{\substack{(u, v) \in K,|u| \leq \tilde{\rho}_{1},|v| \leq \tilde{\rho}_{2} \\\|u\| \leq \varrho_{1},\|v\| \leq \varrho_{2}}}\left\|N_{1}(u, v)\right\| \geq \varrho_{1} \quad \text { or } \inf _{\substack{(u, v) \in K,|u| \leq \tilde{\rho}_{1},|v| \leq \tilde{\rho}_{2} \\\|u\| \leq \varrho_{1},\|v\| \leq \varrho_{2}}}\left\|N_{2}(u, v)\right\| \geq \varrho_{2}
$$

for some $\tilde{\rho}_{1} \leq \rho_{1}, \tilde{\rho}_{2} \leq \rho_{2}$, then we can assume that the solution $\left(u_{1}, v_{1}\right)$ from Theorem 2.12 or 2.13 satisfies $\left\|u_{1}\right\| \geq \varrho_{1}$ or $\left\|v_{1}\right\| \geq \varrho_{2}$ or $\left|u_{1}\right|>\tilde{\rho}_{1}$ or $\left|v_{1}\right|>\tilde{\rho}_{2}$.

Proof. (i) The inequality follows from Theorem 2.9 applied in the case of $r_{i}:=\varrho_{i}$ and $R_{i}:=\rho_{i}$.

(ii) From Theorem 2.4 applied in the case of $r_{i}:=\varrho_{i}, R_{i}:=\rho_{i}$ and

$$
A=\left\{(u, v):\|u\|<\varrho_{1},\|v\|<\varrho_{2},|u| \leq \tilde{\rho}_{1},|v| \leq \tilde{\rho}_{2}\right\},
$$

we obtain that there are no fixed points in $N$ in $A$, which ends the proof.

The next Remark illustrates how Theorem 2.9 can be used to prove the existence of $n$ nontrivial solutions.

Remark 2.15. If $N$ satisfies the conditions of Theorem 2.9 for all pairs of radii

$$
r_{i}^{j} \leq\left\|\phi_{i}\right\|\left\|\chi_{i}\right\| R_{i}^{j} \text { for } i=1,2, j=1,2, \ldots, n,
$$

satisfying

$$
c_{i} R_{i}^{j}<r_{i}^{j+1} \text { for } i=1,2, j=1,2, \ldots, n-1,
$$

then $N$ possesses at least $n$ nontrivial solutions $\left(u_{j}, v_{j}\right)$ with

$$
\left|u_{j}\right| \leq R_{1}^{j},\left|v_{j}\right| \leq R_{2}^{j},\left\|u_{j}\right\| \geq r_{1}^{j},\left\|v_{j}\right\| \geq r_{2}^{j} .
$$

Moreover, if the conditions

$$
\begin{gathered}
\inf _{\substack{(u, v) \in K,|u| \leq r_{1}^{j},|v| \leq r_{2}^{j} \\
\|u\|=r_{1}^{j},\|v\| \geq r_{2}^{j}}}\left\|N_{1}(u, v)\right\|>\frac{r_{1}^{j}}{\left\|\chi_{1}\right\|}, \quad \inf _{\substack{(u, v) \in K,|u| \leq r_{1}^{j},|v| \leq r_{2}^{j} \\
\|u\| \geq r_{1}^{j},\|v\|=r_{2}^{j}}}\left\|N_{2}(u, v)\right\|>\frac{r_{2}^{j}}{\left\|\chi_{2}\right\|}, \\
\sup _{(u, v) \in K,|u| \leq R_{1}^{j},|v| \leq R_{2}^{j}}\left|N_{i}(u, v)\right|<R_{i}^{j} \quad(i=1,2),
\end{gathered}
$$

hold, then we have $n-1$ additional solutions $\left(\bar{u}_{j}, \bar{v}_{j}\right), j=1, \ldots, n-1$ such that

$$
\left|\bar{u}_{j}\right|<R_{1}^{j+1},\left|\bar{v}_{j}\right|<R_{2}^{j+1} ;\left|\bar{u}_{j}\right|>R_{1}^{j} \text { or }\left|\bar{v}_{j}\right|>R_{2}^{j} ;\left\|\bar{u}_{j}\right\|<r_{1}^{j+1} \text { or }\left\|\bar{v}_{j}\right\|<r_{2}^{j+1} .
$$

The first conclusion follows from Theorem 2.9 applied $n$ times, whereas the second follows from Theorem 2.12 applied $n-1$ times.

Remark 2.16. Assume that between the cones $K_{i} \subseteq K_{i}^{0}$ and the norms $|\cdot|_{i}(i=1,2)$ there is a strong compatibility, expressed by the following condition:

(2.36) there exists $h_{i}^{0} \in K_{i},\left|h_{i}^{0}\right|=1$, such that $h_{i}^{0} \geq u$ for every $u \in K_{i}$ with $|u| \leq 1$.

Then the localization theory for fixed points of a given operator $N=\left(N_{1}, N_{2}\right): K \rightarrow K$ can be developed without assuming the Harnack type inequality " $u \geq\|u\| \chi_{i}$ " for the definition of $K$ and without specifying the elements $\chi_{i}, \phi_{i}$. This is based on a different way of proving that the homotopy $H(u, v, t)=t h+(1-t) N(u, v)$ is fixed point free on the boundary. Here we choose $h=\left(R_{1} h_{1}^{0}, R_{2} h_{2}^{0}\right)$. For instance, the corresponding version of Theorem 2.2 is the following: 
Theorem 2.17. Assume that condition (2.36) holds, that $N: K \rightarrow K$ is completely continuous and that for some numbers $0 \leq r_{i}<\left\|h_{i}^{0}\right\| R_{i}$, the conditions

$$
\inf _{\substack{(u, v) \in C \\\|u\|=r_{1},\|v\| \leq r_{2}}}\left\|N_{1}(u, v)\right\|>r_{1}, \quad \inf _{\substack{(u, v) \in C \\\|u\| \leq r_{1},\|v\|=r_{2}}}\left\|N_{2}(u, v)\right\|>r_{2}
$$

and

$$
\sup _{(u, v) \in C}\left|N_{i}(u, v)\right| \leq R_{i} \quad(i=1,2)
$$

hold. Then $N$ has at least one fixed point $(u, v) \in K$ such that $|u| \leq R_{1},|v| \leq R_{2}$ and either $\|u\| \geq r_{1}$ or $\|v\| \geq r_{2}$.

Proof. Following the proof of Theorem 2.2 and assuming that there exists $(u, v) \in \partial U$ and $t \in(0,1)$ with $H(u, v, t)=(u, v)$, in case that $(u, v) \in A_{1}$, we have

$$
u=N_{1}(u, v)+t\left(h-N_{1}(u, v)\right) \geq N_{1}(u, v) .
$$

Therefore, making use of the first inequality from (2.37), we obtain

$$
r_{1}=\|u\| \geq\left\|N_{1}(u, v)\right\|>r_{1},
$$

a contradiction. The same argument can be used if $(u, v) \in A_{2}$. Note that $h \notin \bar{U}$ because of the condition $r_{i}<\left\|h_{i}^{0}\right\| R_{i}(i=1,2)$.

We underline that, although the Harnack inequality is not used in the above proof, it is essential to obtain the estimates from below of the type (2.37) in the applications.

Remark 2.18. We stress that the abstract results obtained in this section can be generalized to the case of systems of more than two equations. The idea is to consider the product space $X=\prod_{i=1}^{n} X_{i}$ of the Banach spaces $X_{i}$, endowed with the norms $|\cdot|_{i}$, seminorms $\|\cdot\|_{i}$, and the cones $K_{i} \subset K_{i}^{0} \subset X_{i}$ such that $\phi_{i} \in K_{i}:=\left\{u \in K_{i}^{0}: u \geq\|u\|_{i} \chi_{i}\right\}$ for fixed $\chi_{i}$ and $\phi_{i}$ satisfying (2.1), $i=1,2, \ldots, n$. In this setting we are interested in the existence and localization of fixed points of a given operator $N: K \rightarrow K$, where $K=\prod_{i=1}^{n} K_{i}$. For example, let us consider the sets

$$
C=\left\{u \in K:\left|u_{1}\right|_{1} \leq R_{1}, \ldots,\left|u_{n}\right|_{n} \leq R_{n}\right\}, U=\left\{u \in C:\left\|u_{1}\right\|_{1}<r_{1}, \ldots,\left\|u_{n}\right\|_{n}<r_{n}\right\}
$$

for given radii $r_{i}, R_{i}$ with $r_{i}<\left\|\phi_{i}\right\|_{i}\left\|\chi_{i}\right\|_{i} R_{i}, i=1, \ldots, n$. If

$$
\sup _{u \in C}\left|N_{i}(u)\right|_{i} \leq R_{i}, \quad i=1,2, \ldots, n
$$

and

$$
\inf _{\substack{(u, v) \in \bar{U} \\\left\|u_{i}\right\|_{i}=r_{i}}}\left\|N_{i}(u)\right\|_{i} \geq \frac{r_{i}}{\left\|\chi_{i}\right\|_{i}}, \quad i=1,2, \ldots, n
$$

then $N$ has at least one fixed point in $C \backslash U$.

As a consequence, results analogous to ones obtained later in Section 3, can be established for systems with more than two differential equations. 
2.4. Case of isotone operators. Let us now turn our attention to the case when $N$ satisfies a monotonicity condition with respect to the order induced by cones $K_{i}^{0}$. Precisely, assume that the operator $N$ is isotone, that is

$$
0 \leq u \leq u^{\prime}, 0 \leq v \leq v^{\prime} \text { implies } N_{1}(u, v) \leq N_{1}\left(u^{\prime}, v^{\prime}\right), N_{2}(u, v) \leq N_{2}\left(u^{\prime}, v^{\prime}\right),
$$

and that condition 2.36 is satisfied.

Let us examine the condition (2.4). If $(u, v) \in C,\|u\|=r_{1},\|v\| \leq r_{2}$, then $u \geq$ $\|u\| \chi_{1}=r_{1} \chi_{1}$ and $\left\|N_{1}(u, v)\right\| \geq\left\|N_{1}\left(r_{1} \chi_{1}, 0\right)\right\|$. Therefore the condition (2.4) is implied by the simpler one:

$$
\left\|N_{1}\left(r_{1} \chi_{1}, 0\right)\right\| \geq \frac{r_{1}}{\left\|\chi_{1}\right\|}, \quad\left\|N_{2}\left(0, r_{2} \chi_{2}\right)\right\| \geq \frac{r_{2}}{\left\|\chi_{2}\right\|} .
$$

Similarly, the condition 2.19 is implied by the following one:

$$
\left\|N_{1}\left(r_{1} \chi_{1}, r_{2} \chi_{2}\right)\right\| \geq \frac{r_{1}}{\left\|\chi_{1}\right\|}, \quad\left\|N_{2}\left(r_{1} \chi_{1}, r_{2} \chi_{2}\right)\right\| \geq \frac{r_{2}}{\left\|\chi_{2}\right\|} .
$$

Let us now examine the condition (2.5). If $|u| \leq R_{1}$ and $|v| \leq R_{2}$ then $u \leq R_{1} h_{1}^{0}$, $v \leq R_{2} h_{2}^{0}$ and $\left|N_{i}(u, v)\right| \leq\left|N_{i}\left(R_{1} h_{1}^{0}, R_{2} h_{2}^{0}\right)\right|, i=1,2$. This shows that the condition

$$
\left|N_{i}\left(R_{1} h_{1}^{0}, R_{2} h_{2}^{0}\right)\right| \leq R_{i} \quad(i=1,2)
$$

implies 2.5 .

\section{Applications to $(p, q)$-Laplacian systems}

3.1. Existence results. We now turn our attention back to the existence and localization of nonnegative weak solutions of the $(p, q)$-Laplace system (1.1). By a nonnegative solution we mean a solution $(u, v)$ with $u \geq 0$ and $v \geq 0$. A nonnegative solution $(u, v)$ is said to be nontrivial if either $u \neq 0$ or $v \neq 0$, and is called positive if both functions $u, v$ are different from zero, equivalently, if $u>0$ and $v>0$.

In order to apply the abstract theorems from the previous section, we choose $X_{1}=$ $X_{2}=L^{\infty}(\Omega)$, and $K_{1}^{0}=K_{2}^{0}=K^{0}:=\left\{u \in L^{\infty}(\Omega): u \geq 0\right\}$. Thus the two norms $|\cdot|_{1},|\cdot|_{2}$ coincide with the usual norm of $L^{\infty}(\Omega)$, simply denoted by $|\cdot|$. Let $S_{r}: L^{\infty}(\Omega) \rightarrow C_{0}^{1}(\bar{\Omega})$ be the operator which assigns to any $v \in L^{\infty}(\Omega)$ the unique weak solution $u$ of the problem

$$
\begin{cases}-\Delta_{r} u=v & \text { in } \Omega, \\ u=0 & \text { on } \partial \Omega,\end{cases}
$$

i.e. $S_{r}=\left(-\Delta_{r}\right)^{-1}$. It is known (see [2]) that $S_{r}$ is well defined, completely continuous, isotone and positive. Also consider the superposition operators $F, G: K^{0} \times K^{0} \rightarrow K^{0}$,

$$
F(u, v)(x)=f(x, u(x), v(x)), \quad G(u, v)(x)=g(x, u(x), v(x)),
$$

and define the operator $N=\left(N_{1}, N_{2}\right)$ by

$$
N_{1}(u, v)=S_{p}(F(u, v)), \quad N_{2}(u, v)=S_{q}(G(u, v)) .
$$

Note that a pair $(u, v)$ is a nonnegative weak solution of 1.1 if and only if it is a fixed point of $N$ in $K^{0} \times K^{0}$. In Remark 3.10 we shall give some additional information on the regularity of the solution. 
We recall a local weak Harnack inequality for nonnegative $p$-superharmonic functions due to N.S. Trudinger, see [21, Theorem 1.2]:

Theorem 3.1. For each $s \in\left[1, \frac{n(p-1)}{n-p}\right)$ if $p<n$ or $s \in[1, \infty)$ if $p \geq n$ and for each $\rho>0$ such that $B_{3 \rho} \subset \Omega$, there exists a constant $C=C(n, p, s)>0$ such that

$$
\inf _{B_{\rho}} u \geq C \rho^{-\frac{n}{s}}\left(\int_{B_{2 \rho}} u^{s} d x\right)^{\frac{1}{s}}
$$

for every nonnegative p-superharmonic function $u$ in $\Omega$.

Following a reasoning based on finite cover by balls of any compact set (see the proof of Corollary 1.2.9 in [15] or Theorem 1.3 in [18]), we obtain a variant of the Harnack inequality, stated in [19], which plays a crucial role in our investigation.

Theorem 3.2. For each $s$ as in Theorem 3.1 and each compact $D \subset \Omega$, there is a constant $M=M(n, p, s, D, \Omega)>0$ such that

$$
\inf _{D} u \geq M\left(\int_{D} u^{s} d x\right)^{\frac{1}{s}}
$$

for every nonnegative p-superharmonic function $u$ in $\Omega$.

By means of the Trudinger-type inequality $(3.2)$ we shall obtain the existence, localization, and multiplicity of nonnegative solutions for the problem (1.1).

Let us now consider any two compact sets $D_{1}, D_{2} \subset \Omega$ and their characteristic functions $\chi_{D_{1}}, \chi_{D_{2}}$, which we denote by $\chi_{1}, \chi_{2}$. Since $p, q>2 n /(n+1)$, by Theorem 3.2 we obtain that there are numbers $s_{1}, s_{1}>1$ and constants $M_{1}, M_{1}>0$ such that

$$
\inf _{D_{1}} u \geq\|u\|_{1}:=M_{1}\left(\int_{D_{1}} u^{s_{1}} d x\right)^{\frac{1}{s_{1}}}, \quad \inf _{D_{2}} v \geq\|v\|_{2}:=M_{2}\left(\int_{D_{2}} u^{s_{2}} d x\right)^{\frac{1}{s_{2}}}
$$

for every nonnegative $p$-superharmonic function $u$ and $q$-superharmionic fuction $v$. Using the natural partial order in $L^{\infty}(\Omega)$ we can rewrite the inequalities $(3.3)$ in the following way:

$$
u \geq\|u\|_{1} \chi_{1}, \quad v \geq\|v\|_{2} \chi_{2}
$$

The nonnegativity of $f$ and $g$ gives that $N_{1}(u, v)$ and $N_{2}(u, v)$ are superharmonic for any $u, v \in K^{0}$. Therefore we obtain in a similar way as in the case of one equation studied in [19], that

$$
N_{1}\left(K^{0} \times K^{0}\right) \subset K_{1}, \quad N_{2}\left(K^{0} \times K^{0}\right) \subset K_{2},
$$

where

$$
K_{i}=\left\{u \in K^{0}: u \geq\|u\|_{i} \chi_{i}\right\}, \quad \chi_{i}=\chi_{D_{i}} \quad(i=1,2) .
$$

Therefore, $N: K_{1} \times K_{2} \rightarrow K_{1} \times K_{2}$. In addition, the complete continuity of $S_{p}$ and $S_{q}$ guarantees that $N$ is completely continuous. 
Remark 3.3. Note that the assumption (2.36) is satisfied in our context of differential equations, since the constant function $h_{i}^{0} \equiv 1$ satisfies 2.36 ). Indeed, since the constant function 1 is $p(q)$-superharmionic in $\Omega$, one has $h_{i}^{0} \in K_{i}$. Also, the conditions $\left|h_{i}^{0}\right|=1$ and $u \leq h_{i}^{0}$ for every $u \in K_{i}$ with $|u| \leq 1$ are trivially satisfied. Therefore we can use Remark 2.16. This yields that, instead of the relation (2.3) between radii, we can consider the relation

$$
r_{i}<\|1\|_{i} R_{i} \quad(i=1,2),
$$

and we can improve the constants $r_{i} /\left\|\chi_{i}\right\|$ in (2.4), (2.13), (2.16), (2.19), (2.33), (2.34), replacing them by $r_{i}$.

Notice that if in our case, $\phi_{i}$ is chosen to be $\chi_{i} /\left|\chi_{i}\right|=\chi_{i}$, then the relation (2.3) becomes $r_{i}<\|1\|_{i}^{2} R_{i}$, which is more restrictive than (3.5).

We can now state a result for the existence and localization of a nonnegative solution of the system 1.1 with both nonzero components (i.e. a positive solution).

Theorem 3.4. Let $r_{1}, r_{2}, R_{1}, R_{2}$ satisfy (3.5). Assume that the following conditions hold:

$$
\begin{aligned}
& \frac{\max _{(x, \tau, \sigma) \in \bar{\Omega} \times\left[0, R_{1}\right] \times\left[0, R_{2}\right]} f(x, \tau, \sigma)}{R_{1}^{p-1}}=: \alpha_{1} \leq A_{p}:=\frac{1}{\left|S_{p}(1)\right|^{p-1}}, \\
& \frac{\max _{(x, \tau, \sigma) \in \bar{\Omega} \times\left[0, R_{1}\right] \times\left[0, R_{2}\right]} g(x, \tau, \sigma)}{R_{2}^{q-1}}=: \alpha_{2} \leq A_{q}:=\frac{1}{\left|S_{q}(1)\right|^{q-1}},
\end{aligned}
$$

and

$$
\begin{aligned}
& \frac{\min _{(x, \tau, \sigma) \in D_{1} \times\left[r_{1}, R_{1}\right] \times\left[0, R_{2}\right]} f(x, \tau, \sigma)}{r_{1}^{p-1}}=: \beta_{1}>B_{1, p}:=\frac{1}{\left\|S_{p}\left(\chi_{1}\right)\right\|^{p-1}}, \\
& \frac{\min _{(x, \tau, \sigma) \in D_{2} \times\left[0, R_{1}\right] \times\left[r_{2}, R_{2}\right]} g(x, \tau, \sigma)}{r_{2}^{q-1}}=: \beta_{2}>B_{2, q}:=\frac{1}{\left\|S_{q}\left(\chi_{2}\right)\right\|^{q-1}} .
\end{aligned}
$$

Then there exists a positive solution $(u, v)$ of (1.1) such that $|u| \leq R_{1},|v| \leq R_{2},\|u\| \geq r_{1}$ and $\|v\| \geq r_{2}$.

Proof. If $(u, v) \in C$ and $x \in \Omega$, then $0 \leq u(x) \leq R_{1}, 0 \leq v(x) \leq R_{2}$ and therefore

$$
F(u, v)(x)=f(x, u(x), v(x)) \leq \alpha_{1} R_{1}^{p-1} .
$$

By the isotonicity of $S_{p}$ and the monotonicity of $|\cdot|$ we have

$$
\left|N_{1}(u, v)\right| \leq\left|S_{p}\left(\alpha_{1} R_{1}^{p-1}\right)\right| \leq \alpha_{1}^{1 /(p-1)} R_{1}\left|S_{p}(1)\right| \leq R_{1} .
$$

A similar estimate is true for $N_{2}$. Hence, the condition 2.20 from Theorem 2.9 is satisfied. Now, let $(u, v) \in C$ be such that $\|u\|=r_{1}$. For $x \in D_{1}$, we have $u(x) \geq\|u\|=r_{1}$ and, as a consequence,

$$
F(u, v)(x)=f(x, u(x), v(x)) \geq \beta_{1} r_{1}^{p-1} .
$$

By the isotonicity of $S_{p}$ and the monotonicity of $\|\cdot\|$ we have

$$
\left\|N_{1}(u, v)\right\| \geq\left\|S_{p}\left(\beta_{1} r_{1}^{p-1} \chi_{1}\right)\right\|_{16}=\beta_{1}{ }^{1 /(p-1)} r_{1}\left\|S_{p}\left(\chi_{1}\right)\right\|>r_{1} .
$$


A similar estimate is true for $N_{2}$. Hence, the condition 2.19 from Theorem 2.9, modified in accordance with Remark 2.16, is satisfied.

The assertion now follows from Theorem 2.9 and Remark 3.3 .

We now present the relationship between the constants that arise in Theorem 3.4 .

Proposition 3.5. Let $\lambda_{1, p}$ be the first eigenvalue of the $p$-Laplace operator $-\Delta_{p}$ under the Dirichlet condition. The following relations hold

$$
A_{p} \leq \lambda_{1, p} \leq B_{1, p} \text { and } A_{q} \leq \lambda_{1, q} \leq B_{2, q}
$$

Proof. Let $u>0$ be a positive eigenfunction corresponding to the eigenvalue $\lambda_{1, p}$. Then $-\Delta_{p} u=\lambda_{1, p} u^{p-1}$ and since $S_{p}$ is homogeneous of degree $1 /(p-1)$, we have

$$
u=S_{p}\left(\lambda_{1, p} u^{p-1}\right)=\lambda_{1, p}^{1 /(p-1)} S_{p}\left(u^{p-1}\right) .
$$

a) Assume that $|u|=1$. Then $0 \leq u \leq 1$ and, by the isotonicity of $S_{p}$ and the monotonicity of the norm, we have

$$
1=|u|=\lambda_{1, p}^{1 /(p-1)}\left|S_{p}\left(u^{p-1}\right)\right| \leq \lambda_{1, p}^{1 /(p-1)}\left|S_{p}(1)\right| .
$$

This implies that $A_{p} \leq \lambda_{1, p}$.

b) Now assume that $\|u\|=1$. Then, by the Harnack inequality, we have $u \geq \chi_{D_{1}}$. By the isotonicity of $S_{p}$ and the monotonicity of the semi-norm we obtain

$$
1=\|u\|=\lambda_{1, p}^{1 /(p-1)}\left\|S_{p}\left(u^{p-1}\right)\right\| \geq \lambda_{1, p}^{1 /(p-1)}\left\|S_{p}\left(\chi_{D_{1}}\right)\right\| .
$$

This implies that $B_{1, p} \geq \lambda_{1, p}$. Similarly one can prove that $A_{q} \leq \lambda_{1, q} \leq B_{2, q}$.

Theorem 3.6. Let $r_{1}, r_{2}, R_{1}, R_{2}$ satisfy 3.5 and let $\tilde{R}_{1} \leq R_{1}, \tilde{R}_{2} \leq R_{2}$. Assume that the condition (3.6) is satisfied and that

$$
\begin{aligned}
& \frac{\min _{(x, \tau, \sigma) \in D_{1} \times\left[0, \tilde{R}_{1}\right] \times\left[0, \tilde{R}_{2}\right]} f(x, \tau, \sigma)}{r_{1}^{p-1}}=: \beta_{1}^{\prime}>B_{1, p} \quad \text { or } \\
& \frac{\min _{(x, \tau, \sigma) \in D_{2} \times\left[0, \tilde{R}_{1}\right] \times\left[0, \tilde{R}_{2}\right]} g(x, \tau, \sigma)}{r_{2}^{q-1}}=: \beta_{2}^{\prime}>B_{2, q} .
\end{aligned}
$$

Then there exists a nontrivial nonnegative solution $(u, v)$ of 1.1 such that $|u| \leq R_{1}$, $|v| \leq R_{2}$ and

$$
\|u\| \geq r_{1} \quad \text { or }\|v\| \geq r_{2} \text { or }|u|>\tilde{R}_{1} \text { or }|v|>\tilde{R}_{2} .
$$

In particular, if $\tilde{R}_{1}=R_{1}$ and $\tilde{R}_{2}=R_{2}$, then there exists a nontrivial nonnegative solution $(u, v)$ with either $\|u\| \geq r_{1}$ or $\|v\| \geq r_{2}$.

Proof. As in the previous proof we know that the condition 2.10 from Theorem 2.4 is satisfied. Let

$$
(u, v) \in A:=\left\{(u, v):\|u\|<r_{1},\|v\|<r_{2},|u| \leq \tilde{R}_{1},|v| \leq \tilde{R}_{2}\right\} .
$$

Assume that the first inequality in $(3.8)$ holds. Then for $x \in D_{1}$ we have

$$
F(u, v)(x)=f(x, u(x), v(x)) \geq \beta_{1}^{\prime} r_{1}^{p-1}
$$


and by the isotonicity of $S_{p}$ and the monotonicity of $\|\cdot\|$,

$$
\left\|N_{1}(u, v)\right\| \geq\left\|S_{p}\left(\beta_{1}^{\prime} r_{1}^{p-1} \chi_{1}\right)\right\|=\beta_{1}^{\prime 1 /(p-1)} r_{1}\left\|S_{p}\left(\chi_{1}\right)\right\|>r_{1} .
$$

A similar estimate holds for $N_{2}$. Hence, the condition 2.11) from Theorem 2.4, modified in accordance with Remark 2.16, is satisfied. Therefore, we can apply Theorem 2.4 and Remark 3.3, and obtain a solution $(u, v) \notin A$. Clearly, this is equivalent to (3.9).

Remark 3.7. The importance of Theorem 3.6 consists in the fact that the assumption (3.8) involves only one component of the system nonlinearity $(f, g)$. Therefore, it allows different kinds of growth of $f$ and $g$ near the origin. A similar remark also applies to the following theorem.

Theorem 3.8. Let $\rho_{i}, r_{i}, R_{i}$ satisfy $0<\rho_{i}<r_{i}<\|1\|_{i} R_{i} \quad(i=1,2)$. Assume that:

$$
\begin{aligned}
& \frac{\max _{(x, \tau, \sigma) \in \bar{\Omega} \times\left[0, R_{1}\right] \times\left[0, R_{2}\right]} f(x, \tau, \sigma)}{R_{1}^{p-1}} \leq A_{p}, \quad \frac{\max _{(x, \tau, \sigma) \in \bar{\Omega} \times\left[0, R_{1}\right] \times\left[0, R_{2}\right]} g(x, \tau, \sigma)}{R_{2}^{q-1}} \leq A_{q}, \\
& \frac{\max _{(x, \tau, \sigma) \in \bar{\Omega} \times\left[0, \rho_{1}\right] \times\left[0, \rho_{2}\right]} f(x, \tau, \sigma)}{\rho_{1}^{p-1}}<A_{p}, \quad \frac{\max _{(x, \tau, \sigma) \in \bar{\Omega} \times\left[0, \rho_{1}\right] \times\left[0, \rho_{2}\right]} g(x, \tau, \sigma)}{\rho_{2}^{q-1}}<A_{q},
\end{aligned}
$$

$$
\frac{\min _{(x, \tau, \sigma) \in D_{1} \times\left[r_{1}, R_{1}\right] \times\left[0, R_{2}\right]} f(x, \tau, \sigma)}{r_{1}^{p-1}}>B_{1, p}, \quad \frac{\min _{(x, \tau, \sigma) \in D_{2} \times\left[0, R_{1}\right] \times\left[r_{2}, R_{2}\right]} g(x, \tau, \sigma)}{r_{2}^{q-1}}>B_{2, q} .
$$

Then there exist three nonnegative solutions $\left(u_{i}, v_{i}\right)(i=1,2,3)$ of the system (1.1) with $\left|u_{1}\right|<\rho_{1}, \quad\left|v_{1}\right|<\rho_{2} \quad$ (possibly zero solution)

$\left\|u_{2}\right\|<r_{1},\left\|v_{2}\right\|<r_{2} ;\left|u_{2}\right|>\rho_{1}$ or $\left|v_{2}\right|>\rho_{2} \quad$ (possibly one solution component zero); $\left\|u_{3}\right\|>r_{1},\left\|v_{3}\right\|>r_{2}$ (both solution components nonzero).

Moreover, having given numbers $0<\varrho_{i}<\|1\| \rho_{i}(i=1,2)$,

(i) if

$$
\frac{\min _{(x, \tau, \sigma) \in D_{1} \times\left[\varrho_{1}, \rho_{1}\right] \times\left[0, \rho_{2}\right]} f(x, \tau, \sigma)}{\varrho_{1}^{p-1}}>B_{1, p} \quad \text { and } \quad \frac{\min _{(x, \tau, \sigma) \in D_{2} \times\left[0, \rho_{1}\right] \times\left[\varrho_{2}, \rho_{2}\right]} g(x, \tau, \sigma)}{\varrho_{2}^{q-1}}>B_{2, q}
$$

then $\left\|u_{1}\right\| \geq \varrho_{1}$ and $\left\|v_{1}\right\| \geq \varrho_{2}$;

(ii) if

$$
\frac{\min _{(x, \tau, \sigma) \in D_{1} \times\left[0, \tilde{\rho}_{1}\right] \times\left[0, \tilde{\rho}_{2}\right]} f(x, \tau, \sigma)}{\varrho_{1}^{p-1}}>B_{1, p} \quad \text { or } \quad \frac{\min _{(x, \tau, \sigma) \in D_{2} \times\left[0, \tilde{\rho}_{1}\right] \times\left[0, \tilde{\rho}_{2}\right]} g(x, \tau, \sigma)}{\varrho_{2}^{q-1}}>B_{2, q}
$$

for some $\tilde{\rho}_{1} \leq \rho_{1}, \tilde{\rho}_{2} \leq \rho_{2}$, then $\left\|u_{1}\right\| \geq \varrho_{1}$ or $\left\|v_{1}\right\| \geq \varrho_{2}$ or $\left|u_{1}\right|>\tilde{\rho}_{1}$ or $\left|v_{1}\right|>\tilde{\rho}_{2}$.

Proof. Observe that 2.2 implies that the constants $c_{i}$ that occur in the statement of the Theorem 2.12 are equal to 1.

The assumptions (3.10), 3.11) and (3.12) imply the conditions 2.31), 2.32, 2.30), modified according to Remark 2.16. Hence, the first part of the assertion follows from Theorem 2.13, combined with Remark 3.3. 
The second part follows, in a similar way, from Theorem 2.14, combined with Remark 3.3 .

Remark 3.9. If the functions $f, g$ do not depend on $x$ and are monotone (increasing or decreasing) in each of the two variables $u, v$, then the minima and maxima in all inequalities from Theorems $3.4+3.8$ are reached on some boundary point of the corresponding rectangular domain. Combining the two kinds of monotonicity on the two variables $u, v$, for our functions $f(u, v), g(u, v)$, we see that sixteen cases are possible. For example, in the case of Theorem 3.4, we may have the following situations:

(1) $f, g$ increasing in both variables; then the conditions (3.6) and (3.7) read as

$$
\begin{array}{ll}
f\left(R_{1}, R_{2}\right) \leq A_{p} R_{1}^{p-1} & g\left(R_{1}, R_{2}\right) \leq A_{q} R_{2}^{q-1} \\
f\left(r_{1}, 0\right)>B_{1, p} r_{1}^{p-1} & g\left(0, r_{2}\right)>B_{2, q} r_{2}^{q-1}
\end{array}
$$

(2) $f$ increasing in $u$ and decreasing in $v$, and $g$ increasing in both $u, v$; then the conditions (3.6) and (3.7) become

$$
\begin{array}{ll}
f\left(R_{1}, 0\right) \leq A_{p} R_{1}^{p-1} & g\left(R_{1}, R_{2}\right) \leq A_{q} R_{2}^{q-1} \\
f\left(r_{1}, R_{2}\right)>B_{1, p} r_{1}^{p-1} & g\left(0, r_{2}\right)>B_{2, q} r_{2}^{q-1} .
\end{array}
$$

Remark 3.10. It is worth mentioning that the solutions $(u, v)$ of the system (1.1) are continuously differentiable. This follows from the fact that $S_{r}\left(L^{\infty}(\Omega)\right) \subset C^{1}(\bar{\Omega}), r>1$ (see [2]). Moreover, as a consequence of the Harnack inequality (see [21]), if $u \geq 0$ and $u \neq 0$, then

$$
u(x)>0 \text { for } x \in \Omega
$$

(the same for $v$ ). Indeed, the Harnack inequality implies that the set $\{x \in \Omega: u(x)=0\}$ is open. Being also closed in $\Omega$, it is equal either to $\emptyset$ or to $\Omega$. The latter is excluded by the assumption $u \neq 0$.

3.2. Non-existence results. We now present some sufficient conditions for the nonexistence of positive solutions of the system (1.1).

Theorem 3.11. Assume that $(u, v)$ is a nonnegative solution of the system (1.1). If one of the following conditions holds:

$$
\begin{aligned}
& f(x, u, v)<\lambda_{1, p} u^{p-1}, \text { for every }(x, u, v) \in \Omega \times(0, \infty) \times[0, \infty), \\
& f(x, u, v)>\lambda_{1, p} u^{p-1}, \text { for every }(x, u, v) \in \Omega \times(0, \infty) \times[0, \infty), \\
& f(x, u, v)>B_{1, p} u^{p-1}, \text { for every }(x, u, v) \in D_{1} \times(0, \infty) \times[0, \infty),
\end{aligned}
$$

then $u=0$. Similarly, if one of the following conditions holds:

$$
\begin{aligned}
& g(x, u, v)<\lambda_{1, q} v^{q-1}, \text { for every }(x, u, v) \in \Omega \times[0, \infty) \times(0, \infty), \\
& g(x, u, v)>\lambda_{1, q} v^{q-1}, \text { for every }(x, u, v) \in \Omega \times[0, \infty) \times(0, \infty), \\
& g(x, u, v)>B_{2, q} v^{q-1}, \text { for every }(x, u, v) \in D_{2} \times[0, \infty) \times(0, \infty),
\end{aligned}
$$

then $v=0$. 
Proof. Let us observe that $-\Delta_{p} u=f(x, u, v) \geq 0$. Suppose on the contrary that $u \neq 0$. Then $u(x)>0$ for $x \in \Omega$ (see Remark 3.10).

Assume that the inequality (3.15) holds. Then $f(x, u(x), v(x))<\lambda_{1, p} u^{p-1}(x)$ almost everywhere in $\Omega$ and consequently

$$
\lambda_{1, p} \int_{\Omega} u^{p} \leq \int_{\Omega}|\nabla u|^{p}=\int_{\Omega} f(x, u, v) \cdot u<\lambda_{1, p} \int_{\Omega} u^{p-1} u,
$$

a contradiction.

Assume now that 3.16 holds. Then

$$
-\Delta_{p} u=\lambda_{1, p} u^{p-1}+h,
$$

where the function $h(x):=f(x, u(x), v(x))-\lambda_{1, p} u^{p-1}(x)$ is positive almost everywhere in $\Omega$ and is obviously in the space $L^{\infty}(\Omega)$. This contradicts the fact from [8], which states that (3.21) has no solutions.

Assume now that the inequality (3.17) holds. Put $r:=\|u\|$. Then, since $u \in K_{1}$, we have that $u(x) \geq r$ for $x \in D_{1}$. Therefore

$$
F(u, v)(x)=f(x, u(x), v(x))>B_{1, p} u(x)^{p-1} \geq B_{1, p} r^{p-1} .
$$

By the continuity of $f, u$ and $v$ (see Remark 3.10) and the compactness of $D_{1}$ we deduce that $F(u, v)(x) \geq B_{1, p} r_{1}^{p-1}$ for some $r_{1}>r$ and all $x \in D_{1}$. This implies that $F(u, v) \geq$ $B_{1, p} r_{1}^{p-1} \chi_{1}$. Then, by the isotonicity of $S_{p}$, the monotonicity of the seminorm and the definition of $B_{1, p}$, we derive

$$
r=\|u\|=\left\|N_{1}(u, v)\right\|=\left\|S_{p} F(u, v)\right\| \geq\left\|S_{p}\left(B_{1, p} r_{1}^{p-1}\right)\right\|=B_{1, p}^{1 /(p-1)} r_{1}\left\|S_{p}\left(\chi_{1}\right)\right\|=r_{1},
$$

a contradiction.

The second assertion can be proved analogously.

Corollary 3.12. (i) If one of the inequalities (3.15)-3.20 holds, then there are no positive solutions of the system (1.1).

(ii) If one of the inequalities (3.15)-(3.17) holds and one of the inequalities (3.18)-(3.20) holds, then there are no nontrivial nonnegative solutions of the system (1.1).

\subsection{Example. Let}

$$
f(x, u, v)=\varphi(x, u, v) \cdot \frac{u^{2}}{4+u^{3}}, \quad g(x, u, v)=\psi(x, u, v) \cdot \arctan ^{2} v
$$

where $\varphi$ and $\psi$ are continuous functions such that

$$
a \leq \varphi(x, u, v) \leq b, \quad c \leq \psi(x, u, v) \leq d,
$$

for every $(x, u, v) \in \Omega \times[0,+\infty) \times[0,+\infty)$ and some fixed constants $a, b, c, d \in(0,+\infty)$.

Proposition 3.13. Under the above assumptions, there exists a constant $\lambda_{0}$ (depending on $a, b, c, d$ and $\Omega$ ) such that for each $\lambda>\lambda_{0}$, the problem

$$
\begin{cases}-\Delta u=\lambda f(x, u, v) & \text { in } \Omega, \\ -\Delta v=\lambda g(x, u, v) & \text { in } \Omega, \\ u, v=0 & \text { on } \partial \Omega,\end{cases}
$$

has at least two nontrivial nonnegative solutions. 
Proof. We shall apply Theorem 3.8 . To this end, consider any compact subset $D \subset \Omega$ and put $D_{1}:=D, D_{2}:=D$. In order to simplify our notation we denote

$$
A:=A_{2}, B:=B_{1,2}=B_{2,2}, \Phi(x)=x^{2} /\left(4+x^{3}\right) \text { and } \Psi(x)=\arctan ^{2} x .
$$

Note that $\Phi(x) \leq l_{1}:=1 / 3, \Psi(x) \leq l_{2}:=\pi^{2} / 4, \Psi$ is increasing, while $\Phi$ is increasing in $[0,2]$ and decreasing in $[2, \infty)$. Therefore, if $\rho_{1}<2$ then the conditions (3.10), (3.11) and (3.12) are implied by the following ones:

$$
\begin{gathered}
\lambda l_{1} b \leq A R_{1}, \quad \lambda l_{2} d \leq A R_{2}, \\
\frac{\Phi\left(\rho_{1}\right)}{\rho_{1}}<\frac{A}{\lambda b}, \quad \frac{\Psi\left(\rho_{2}\right)}{\rho_{2}}<\frac{A}{\lambda d}, \\
\lambda a \min \left\{\Phi\left(r_{1}\right), \Phi\left(R_{1}\right)\right\}>B r_{1}, \quad \frac{\Psi\left(r_{2}\right)}{r_{2}}>\frac{B}{\lambda c} .
\end{gathered}
$$

If we put

$$
R_{1}(\lambda):=\lambda l_{1} b / A \quad \text { and } \quad R_{2}(\lambda):=\lambda l_{2} d / A,
$$

then the condition 3.23 is satisfied and the condition 3.25 has the form

$$
\frac{R_{1}(\lambda)}{r_{1}} \min \left\{\Phi\left(r_{1}\right), \quad \Phi\left(R_{1}\right)\right\}>l_{1} \frac{B}{A} \frac{b}{a}=: \gamma, \frac{\Psi\left(r_{2}\right)}{r_{2}}>\frac{B}{\lambda c} .
$$

Fix $r_{2}>0$. There exists $\lambda_{1}$ such that the second inequality from (3.26), as well as $r_{2}<\|1\| R_{2}(\lambda)$, are satisfied for $\lambda>\lambda_{1}$. Let us put

$$
r_{1}=r_{1}(\lambda)=1 / \sqrt{R_{1}(\lambda)}
$$

Then there exists $\lambda_{2} \geq \lambda_{1}$ such that $r_{1}(\lambda)<\|1\| R_{1}(\lambda)$ for $\lambda>\lambda_{2}$. The first inequality from 3.26 becomes

$$
\min \left\{\frac{R_{1}(\lambda) \sqrt{R_{1}(\lambda)}}{1+4 R_{1}(\lambda) \sqrt{R_{1}(\lambda)}}, \frac{R_{1}^{3}(\lambda)}{4+R_{1}^{3}(\lambda)}\right\}>\frac{\gamma}{\sqrt{R_{1}(\lambda)}} .
$$

If $\lambda \rightarrow \infty$, then the left-hand side of (3.27) tends to $1 / 4$ and the right-hand side of (3.27) tends to 0 . Therefore, there exists $\lambda_{0} \geq \lambda_{2}$ such that (3.27) is satisfied for $\lambda>\lambda_{0}$. Having defined $r_{i}$ and $R_{i}$ for $\lambda>\lambda_{0}$, let us choose $\rho_{1}<r_{1}$ and $\rho_{2}<r_{2}$ that satisfy (3.24). This is possible because $\Phi(x)=o(x)$ and $\Psi(x)=o(x)$ in 0. By Theorem 3.8 we obtain the existence of at least two nontrivial nonnegative solutions to 3.22 for $\lambda>\lambda_{0}$.

Let us observe that for a fixed $\lambda>\lambda_{0}$, the nontrivial nonnegative solutions $\left(u_{1}, v_{1}\right)$ and $\left(u_{2}, v_{2}\right)$ given by Theorem 3.8 , satisfy

$$
\begin{aligned}
\left|u_{i}\right| & \leq R_{1}(\lambda),\left|v_{i}\right| \leq R_{2}(\lambda) \quad(i=1,2),\left\|u_{1}\right\|>r_{1}(\lambda),\left\|v_{1}\right\|>r_{2}, \\
\left\|u_{2}\right\| & <r_{1}(\lambda),\left\|v_{2}\right\|<r_{2} \text { and }\left|u_{2}\right|>\rho_{1}(\lambda) \text { or }\left|v_{2}\right|>\rho_{2}(\lambda) .
\end{aligned}
$$

Note that both components of the first solution are positive. 


\section{ACKNOWLEDGments}

R. Precup was supported by a grant of the Romanian National Authority for Scientific Research, CNCS - UEFISCDI, project number PN-II-ID-PCE-2011-3-0094. This paper was written during the postdoctoral stage of M. Maciejewski at the University of Calabria, supported by a research fellowship within the project "Enhancing Educational Potential of Nicolaus Copernicus University in the Disciplines of Mathematical and Natural Sciences" (Project no. POKL.04.01.01-00-081/10). A support from the Chair of Nonlinear Mathematical Analysis and Topology is gratefully acknowledged.

\section{REFERENCES}

[1] A. Aghajani and J. Shamshiri. Multiplicity of positive solutions for quasilinear elliptic $p$-Laplacian systems. Electron. J. Differential Equations, No. 111: 1-16, 2012.

[2] C. Azizieh and Ph. Clément. A priori estimates and continuation methods for positive solutions of p-Laplace equations. J. Differential Equations, 179:213-245, 2002.

[3] C. Azizieh, Ph. Clément, and E. Mitidieri. Existence and a priori estimates for positive solutions of p-Laplace systems. J. Differential Equations, 184(2):422-442, 2002.

[4] Ph. Clément, J. Fleckinger, E. Mitidieri and F. de Thélin. Existence of positive solutions for a nonvariational quasilinear elliptic system. J. Differential Equations, 166(2):455-477, 2000.

[5] Ph. Clément, M. García-Huidobro, I. Guerra and R. Manásevich. On regions of existence and nonexistence of solutions for a system of p-q-Laplacians. Asymptot. Anal., 48(1-2):1-18, 2006.

[6] K. Deimling. Nonlinear Functional Analysis, Springer, Berlin, 1985.

[7] S. El Manouni, K. Perera and R. Shivaji. On singular quasi-monotone $(p, q)$-Laplacian systems. Proc. Roy. Soc. Edinburgh Sect. A, 142(3):585-594, 2012.

[8] J. Fleckinger, J.-P. Gossez, P. Takáč and F. de Thélin. Existence, nonexistence et principe de l'antimaximum pour le p-laplacien. C. R. Acad. Sci. Paris Sér. I Math., 321(6):731-734, 1995.

[9] J. Giacomoni, J. Hernández and A. Moussaoui. Quasilinear and singular systems: the cooperative case. In Nonlinear elliptic partial differential equations, volume 540 of Contemp. Math., pages 79-94. Amer. Math. Soc., Providence, RI, 2011.

[10] A. Granas and J. Dugundji. Fixed Point Theory, Springer, New York, 2003.

[11] D. D. Hai and R. Shivaji. An existence result on positive solutions for a class of $p$-Laplacian systems. Nonlinear Anal., 56(7):1007-1010, 2004.

[12] D. D. Hai and H. Wang. Nontrivial solutions for p-Laplacian systems. J. Math. Anal. Appl., 330(1):186-194, 2007.

[13] G. Infante and P. Pietramala. Existence and multiplicity of non-negative solutions for systems of perturbed Hammerstein integral equations. Nonlinear Anal., 71:1301-1310, 2009.

[14] P. Jebelean and R. Precup. Solvability of $p, q$-Laplacian systems with potential boundary conditions. Appl. Anal., 89(2):221-228, 2010.

[15] J. Jost. Partial Differential Equations, Springer-Verlag, New York, 2002.

[16] K. Q. Lan and Z. Zhang. Nonzero positive weak solutions of systems of $p$-Laplace equations. $J$. Math. Anal. Appl., 394(2):581-591, 2012.

[17] L. Montoro, B. Sciunzi, and M. Squassina. Symmetry results for nonvariational quasi-linear elliptic systems. Adv. Nonlinear Stud., 10(4):939-955, 2010.

[18] R. Precup. Moser-Harnack inequality, Krasnosel'skii type fixed point theorems in cones and elliptic problems. Topol. Methods Nonlinear Anal., 40(2):301-313, 2012.

[19] R. Precup. Abstract weak Harnack inequality, multiple fixed points and $p$-Laplace equations. $J$. Fixed Point Theory Appl., 12:193-206, 2012.

[20] Y. Shen and J. Zhang. Multiplicity of positive solutions for a semilinear $p$-Laplacian system with Sobolev critical exponent. Nonlinear Anal., 74(4):1019-1030, 2011. 
[21] N. S. Trudinger. On Harnack type inequalities and their applications to quasilinear elliptic equations. Comm. Pure Appl. Math., 20:721-747, 1967.

[22] H. Wang. Existence and nonexistence of positive radial solutions for quasilinear systems. Discrete Contin. Dyn. Syst., (Dynamical Systems, Differential Equations and Applications. 7th AIMS Conference, suppl.):810-817, 2009.

[23] L. Wei and Z. Feng. Existence and nonexistence of solutions for quasilinear elliptic systems. Dyn. Partial Differ. Equ., 10(1):25-42, 2013.

Gennaro Infante, Dipartimento di Matematica ed Informatica, Università della Calabria, 87036 Arcavacata di Rende, Cosenza, Italy

E-mail address: gennaro.infante@unical.it

Mateusz Maciejewski, Nicolaus Copernicus University, Faculty of Mathematics and Computer Science, ul. Chopina 12/18, 87-100 Toruń, Poland

E-mail address: Mateusz.Maciejewski@mat.umk.pl

Radu Precup, Departamentul de Matematică, Universitatea Babeş-Bolyai, Cluj 400084, ROMANIA

E-mail address: r.precup@math.ubbcluj.ro 